\title{
SENTIMENTOS PRESENTES NAS MULHERES DIANTE DA PERDA FETAL: UMA REVISÃO
}

\author{
Claudia Aparecida Marchetti Duarte* \\ Egberto Ribeiro Turato
}

\begin{abstract}
RESUMO. O interesse pelo tema surgiu a partir de uma pesquisa qualitativa de campo ligada a uma dissertação de mestrado atualmente em desenvolvimento junto ao Programa de Pós-Graduação em Tocoginecologia da Faculdade de Ciências Médicas/Unicamp. Para obtenção dos artigos realizou-se um levantamento bibliográfico junto ao Medline, ao PsycInfo e ao SciELO, utilizando-se as palavraschave fetal loss, stillbirth, grief, meanings, qualitative e research. Também foram considerados capítulos de livros de autores consagrados na área. Os artigos encontrados nas bases de dados, em consonância com os autores consagrados na área, mostram que as experiências de mulheres que sofreram perda fetal evidenciam sentimentos complexos, os quais trazem à tona conflitos relacionados à identidade feminina, ao papel da mulher na sociedade e à morte e seus tabus. O trabalho evidencia também a importância de a equipe de saúde conhecer a dinâmica emocional das mulheres que vivenciaram a experiência de perda.
\end{abstract}

Palavras-chave: Óbito fetal, saúde da mulher, acontecimentos que mudam a vida.

\section{WOMEN'S FEELINGS IN THE FACE OF FETAL LOSS: A REVIEW}

\begin{abstract}
The interest about the subject came from a qualitative field research joined to dissertation of master degree in development together with Pos-Graduation Program in Tocogynecology of the Medical Sciences College of the University of Campinas (UNICAMP). The articles were obtained through a bibliographic survey which was realized in the Medline, PsyInfo and SciELO, using the following keywords: "fetal loss", "stillbirth", "grief", "meanings", "qualitative research". Chapters of book of consecrated authors in this area were also considered. The articles found in the database, in consonance with the consecrated authors in the field, present us that the women experiences who suffered a fetal loss evidence complex feelings, because these feelings bring out conflicts related to feminine identity, woman role in the society, death and its taboo. The importance of the health team to realize the emotional dynamics of women who have lived the loss experience is also evidenced.
\end{abstract}

Key words: Stillbirth, women's health, events that change one's life.

\section{SENTIMIENTOS PRESENTES EN LAS MUJERES FRENTE A LA PÉRDIDA FETAL: UNA REVISIÓN}

RESUMEN. El interés sobre el tema surgió a partir de una investigación cualitativa de campo ligada a la disertación de la maestría (magíster), actualmente en desarrollo, del Programa de Postgrado en Tocoginecología de la Facultad de Ciencias Médicas/ Unicamp. Para la obtención de los artículos ha sido realizada una búsqueda bibliográfica en las bases de datos Medline, PsycInfo y SciELO, utilizándose las siguientes palabras clave: "fetal loss", "stillbirth", "grief", "meanings" y "qualitative research". También han sido considerados capítulos de libros de autores reconocidos. Los artículos encontrados en las bases de datos, añandido a los autores en este área, nos enseñan que las experiencias de las mujeres que han sufrido una pérdida fetal evidencian sentimientos complejos, pues éstos revelan conflictos relacionados a la identidad femenina, al rol de la mujer en la sociedad, a la muerte y sus tabúes. También se destaca la importancia de que el equipo de salud conozca la dinámica emocional de las mujeres que han vivido la experiencia de pérdida.

Palabras-clave: Muerte fetal, salud de la mujer, acontecimientos que cambian la vida.

Este texto propõe-se a discutir alguns dos sentimentos mais frequentemente despertados na mulher após a morte de um bebê antes de seu nascimento. Tal discussão será feita através de artigos científicos encontrados nas bases de dados on-line, nas quais os dados relatados foram obtidos por meio de

\footnotetext{
* Psicóloga, mestranda do Programa de Pós-Graduação em Tocoginecologia, da Faculdade de Ciências Médicas da UNiversidade de Campinas-Unicamp.

\# Médico. Psiquiatra. Docente e orientador, Coordenador do LPCQ -Laboratório de Pesquisa Clínico-Qualitativa.
} 
uma metodologia de pesquisa qualitativa. Não ficaram fora desta discussão as opiniões de autores consagrados a respeito do tema.

Mariani Neto e Aquino (2000) referem que a obstetrícia, enquanto especialidade médica, considera como óbito fetal a morte do feto a partir de $20^{\mathrm{a}}$ semana de idade gestacional, sendo a morte do feto ocorrida anteriormente à sua expulsão ou extração. Segundo conceitos da literatura, o óbito fetal precoce é aquele que ocorre entre 20 e 28 semanas de idade gestacional, enquanto o tardio sucede após 28 semanas completas. De acordo com Fretts (2005), existem vários fatores associadas ao óbito fetal, que incluem doenças maternas, malformações fetais, infecções adquiridas na gestação e alterações placentárias ou no desenvolvimento fetal.

Sabemos, seja inicialmente pela observação de casos na vivência clinica enquanto profissionais de saúde, seja pela eventual vivência empírica partilhada com pessoas de nossos círculos de relações sociais que a morte de um filho antes de seu nascimento costuma representar certa impossibilidade de transcendência de expectativas naturais humanas. Também especificamente interrompe as esperas existenciais depositadas nesse projeto particular pertencente à vida da maternidade.

Sendo assim, a elaboração do luto pela morte de uma criança antes de seu nascimento tem uma dinâmica diferente. Para Defey (1992), nesse processo de elaboração pela perda da criança, depois de ocorrido o parto, é comum uma sensação na mulher de estar vivendo algo como um sonho. Por não ter a criança aninhada em seus braços para prestar-lhe os cuidados necessários, como havia imaginado, ela não se sente mãe da criança, daí a sensação de despersonalização. Registra-se também uma vontade forte de rememorar, repetidamente, o acontecimento em seus detalhes, talvez como se fosse possível voltar no tempo, "apagando" da memória tudo o que de ruim aconteceu e mudar o rumo da experiência. Assim sendo, a angústia pela morte do bebê se expressa por aquilo que podemos denominar de repetidas provas de realidade. É nesta fase que pode manifestar-se uma depressão clínica, o que não raramente mobiliza ainda mais os familiares e também médicos e enfermeiros. É importante que a mulher possa vivenciar este momento de modo - digamos - lúcido, para facilitar a reorganização emocional, integrando à sua vida aquela perda real como se fizesse parte de sua própria história vital.

\section{OBJETIVO}

O objetivo do presente trabalho é realizar uma revisão da literatura de periódicos, em datas-bases on- line, de língua inglesa e portuguesa, entre 2001 e 2007, e de livros de textos usuais na área, sobre os sentimentos mais frequentemente despertados na mulher após a morte de um bebê antes de seu nascimento, visando a uma pesquisa qualitativa de campo.

\section{RECURSOS METODOLÓGICOS}

Para identificarmos e conhecermos o teor de estudos sobre a (des)organização emocional e vital provocada em mulheres após a ocorrência do óbito de seu bebê antes do nascimento, realizamos um levantamento bibliográfico junto às bases mais reconhecidas no meio acadêmico, como Medline, PsycInfo e SciELO. Para tanto, os autores elegeram como palavras-chave as expressões fetal loss, stillbirth, grief, meanings e qualitative research, cruzando-as de modo a elencar abstracts pertinentes ao assunto de nosso interesse. Adicionalmente, foram considerados capítulos de livros de alguns autores habitualmente utilizados como referência teórica por parte dos profissionais na área, tais como Bartilotti, Soifer, Maldonado, Defey, Quayle, Klaus e Kennel, Videla e Grieco. Ainda quanto aos artigos científicos, foram selecionados aqueles cujo método de pesquisa utilizado tenha sido o recurso qualitativo, objetivandose que os resultados levantados pudessem, em um momento posterior, ser utilizados para ancoragem e cotejamento da investigação empreendida em serviço de saúde a que se vinculam os autores evidentemente, desde que os trabalhos separados para análise tivessem como foco central o tema psicológico da morte de bebê antes do nascimento e/ou uma discussão sobre sentimentos adversos, como angústias clínicas provocadas na mulher que vivenciou a perda.

Após os procedimentos aqui descritos, foi identificada uma amostra de 26 (vinte e seis) artigos que englobavam o tema óbito fetal estudado sob diferentes métodos de pesquisa. Foram selecionados, então, sete artigos, em atenção aos critérios de inclusão estipulados. Fique claro que, por opção metodológica, foram excluídos os artigos que focalizaram sentimentos de angústias presentes nos genitores masculinos, em pessoas da família em geral e na equipe de saúde, ante a ocorrência da perda fetal. Também foram excluídos os artigos que usaram recursos de metodologias quantitativas de pesquisa, tais como os de caráter epidemiológico, haja vista que seus resultados constituem-se em conclusões matematicizadas, cuja anteposição a resultados de uma futura pesquisa qualitativa não faz coadunação com o 
rigor técnico-conceitual. Três artigos foram excluídos por tratarem de revisão de literatura.

\section{RESULTADOS E DISCUSSÃO}

Para melhor organização dos dados da revisão de literatura, optamos por iniciar a apresentação e debate com as concepções fundamentais do tema aqui em foco, normalmente ponto de partida de entendimento científico do problema, o que esperadamente se encontra em publicações de livros-texto. Serão apresentados, mais abaixo, aqueles artigos extraídos dos periódicos eletrônicos disponibilizados.

Diante da perda da criança, Quayle (1997) refere que a contínua construção da identidade de mulher grávida, desenvolvida delicadamente ao longo da gestação, sofre uma brusca interrupção. A mulher tem, então, que lidar com sentimentos de impotência, de incapacidade, causando grande impacto em sua feminilidade.

Dessa forma, segundo Bartilotti (1998), rompe-se a possibilidade do exercício da maternidade, o que traz à tona o sentimento de fracasso. Soifer (1992) comenta ainda que os desejos e sonhos da mulher em relação àquela criança são frustrados, impossibilitando-a de utilizar sua capacidade maternal e trazendo-lhe uma dor insuportável.

De acordo com Defey (1992), é compreensível que sentimentos de inveja - perceptíveis em si ou não conscientes - em relação às mulheres que tiveram filhos viventes e saudáveis também possam emergir e trazer consequências psicológicas de difícil manejo emocional. A mulher pode sentir-se tal como uma depositária de coisas muito temidas na vida das pessoas: a doença que limita as potencialidades humanas, a loucura que aliena a autonomia enquanto ser humano e, por fim, a morte como evento irreversível de fechamento do ciclo vital. Reconhecidos sentimentos de culpa também devem ser mencionados, geralmente acompanhados de certa necessidade psicológica de entender as "causas" da perda fetal ocorrida, pois o que permanece no campo do desconhecido e do obscuro frequentemente fragiliza o mundo psíquico interno e os manejos necessários das demandas implacáveis do mundo externo, mesmo aquelas exigências do cotidiano.

Quanto à questão da indução clínica do parto, Quayle (1997) comenta que não é rara, nesta fase, a existência de fantasias de contaminação pela morte, já que a mãe vivente e o filho morrente ainda formam um continuum biológico. Nestas condições, o trabalho de parto é, geralmente, vivenciado como muito doloroso em todos os sentidos, fazendo as angústias pela perda da criança serem expressas somatoriamente à dor fisiológica do trabalho de parto.

Essa vivência complexa, de acordo com Videla e Grieco (1993), implica numa forma única de elaboração do luto pela morte da criança. A construção de vínculos afetivos fortes $\mathrm{e}$ de recordações de convivência mútua fica impossibilitada, uma vez que lembranças não podem ser evocadas posteriormente e a ausência da criança é profundamente sentida, como se fosse retirada parte do corpo. Essa ausência de lembranças também pode trazer a sensação de que a criança foi alguém que não existiu.

Sendo assim, Klaus e Kennell (1992) sugerem que a mulher e seu companheiro sejam encorajados a olhar o bebê e, se possível, tocá-lo. Essa vivência pode ser considerada pelos pais como uma experiência extremamente dolorosa e despertar, a princípio, uma tristeza profunda; porém, para Maldonado (1982), esse momento marca a realidade da perda.

É licito, ainda, pontuarmos que nesse período é comum certo afastamento social, chegando a um ensimesmamento. Para Defey (1992), merecem destaque as experiências de processos diversos de somatização, provavelmente como uma forma de expressão das angústias por via corporal. Ideações hipocondríacas também estão encontradas nesses casos, e partimos da premissa de que imaginarem-se fracamente doentes demonstra dificuldades de separação entre ser - ou ter sido - mãe e o bebê morto, entre a doença e a saúde. Particular atenção merece também outro fenômeno preocupante: o desejo de morrer junto com o filho, que pode parecer, ainda que sutilmente, fantasias de atos de suicídio ou sensação de estar enlouquecendo .

Nos artigos extraídos dos periódicos eletrônicos encontramos um estudo feito na Indonésia por Andajani-Suthahjo e Manderson (2004) que apresenta entrevistas em profundidade realizadas com dez mulheres, entre oito e dez semanas após o parto, com o objetivo de focalizar as experiências de puérperas cujos bebês morrem nas primeiras semanas de vida ou nas quais houve diagnóstico de óbito fetal na gestação. De acordo com os autores, as mulheres demonstraramse confusas em relação à causa da perda fetal ou da morte do bebê, quando questionadas a respeito do que havia acontecido. Reportavam-se à falta de experiência por vivenciarem a primeira experiência como mães. Oito das mulheres relataram não ter recebido informações adequadas, apesar de afirmarem ter desejado recebê-las. A falta de informações claras levou a fantasias a respeito da causa da perda e sentimento de culpa, por acreditar que elas fossem 
ignorantes, negligentes ou incompetentes. Houve também a crença de que a perda tivesse advindo como consequência de um ato mau por elas cometido no passado. Algumas mulheres relataram ter sido acusadas de falta de cuidado adequado por familiares, vizinhos ou mesmo profissionais de saúde. Também relataram isolamento social e mudança radical na rotina de vida.

Em estudo qualitativo realizado na Austrália por St John, Cooke e Goopy (2006), com três mulheres que vivenciaram a perda fetal, descrevem-se resultados interessantes. As mulheres do estudo redigiram minibiografias e foram submetidas a entrevistas em profundidade, realizadas em suas próprias casas. Ao contarem suas histórias, as mulheres desse estudo evidenciaram a necessidade de mudanças nas práticas de saúde e de suporte mais adequado às mulheres que perderam seus bebês antes de seu nascimento, não somente no puerpério, mas também nas gestações subsequentes. Referem ser de extrema importância ter alguém com quem conversar, pois se o houvessem tido não teriam se sentido isoladas e desamparadas. Consideram que, nas gestações subsequentes, ter esse apoio profissional é importante para lidarem com as ansiedades relacionadas à gestação e à perda gestacional anteriormente ocorrida. Além disso, sugeriram cuidados pré-natais específicos para mulheres que vivenciaram perdas gestacionais anteriores, através de trabalho em grupo com profissionais da área. Dessa forma, encontrariam espaço para falar de seus sentimentos e vivenciar a gestação com certo orgulho, e não com medo paralisante.

Hsu, Tseng, Banks e Kuo (2004) realizaram uma pesquisa com 20 mulheres de Taiwan, com o objetivo de explorar os significados que elas haviam atribuído ao óbito fetal, dentro daquele contexto sociocultural específico. Nessa cultura, a morte é vivenciada como um tabu e o bebê que nasceu morto não é reconhecido como um bebê real.Assim sendo,a perda de uma criança após uma gestação longa e desejada torna-se um grande desafio para as mulheres que experimentaram a perde fetal após diagnosticado o óbito.

Ainda sobre o artigo citado acima, é importante destacar que as entrevistas realizadas com as mulheres, ao longo de dois anos e meio, foram interpretadas por meio de análise temática, em que vários temas foram levantados. Dentro de tais temas, os sentimentos presentes foram: perda de controle, quebra de sonhos, sentimento de incompletude, culpa, derrota pessoal, sentimento de menos-valia como mulher e em relação ao seu papel na sociedade. Os autores referem que tabus culturais relacionados à morte, à participação nos rituais de morte e à dificuldade de expressar publicamente seus sentimentos afetam as mulheres de Taiwan na elaboração adequada do luto pela morte do bebê. Para tanto, seria necessário que a mulher e sua família fossem incentivadas pela equipe de saúde a expressar seus sentimentos em relação à morte e a participar dos rituais de despedida da criança para que a elaboração dos sentimentos relacionados ao enlutamento ocorresse adequadamente.

Ainda mencionando o estudo citado, os autores escreveram outro artigo (Hsu et al., 2002) enfocando a maneira como as mulheres de Taiwan transformam os sentimentos relacionados ao enlutamento. Os autores referem que, por causa do tabu relacionado a não falar sobre a morte, a maior parte das mulheres de Taiwan não consegue utilizar-se as mesmas estratégias de enfrentamento do luto, se comparadas às mulheres do Ocidente. Os autores enfocam também a possibilidade de ver o bebê, batizá-lo a até nomeá-lo, ações que podem auxiliar as mulheres na elaboração nãopatológica do luto.

Sobre o referido estudo, vale destacar que as mulheres descreveram seus filhos mortos antes do nascimento como fantasmas que precisam de rituais religiosos para proteção - rituais esses comuns da religião do país. Tais rituais são aceitos pela sociedade e permitem o reconhecimento público do óbito do bebê. Tal atitude ajuda aquelas mulheres a lidar abertamente com o luto e com eventuais culpas relacionadas à perda fetal ocorrida. Outra estratégia utilizada pelas mulheres é a antecipação de nova gestação como forma de elaboração do luto e como maneira de corresponder à expectativa cultural de continuidade da linhagem familiar do marido. Além disso, nesses casos é comum certa fantasia de que outra gestação fará com que se sintam capazes de gerar filhos saudáveis e readquirir a sensação de também serem saudáveis física e emocionalmente. É importante mencionar, ainda, uma afirmação dos autores de que elaborar a perda de um bebê que morre antes de nascer é uma questão de saúde, mas também uma questão de adaptação sociocultural.

Outro artigo da literatura recente relata uma pesquisa realizada por Trulson e Radestad (2004) referente à importância dos cuidados recebidos pela mulher para o seu bem-estar emocional durante o parto de um bebê que morre antes de nascer. No estudo, vinte mulheres foram entrevistadas e falaram a respeito de sua experiência antes e depois do diagnóstico de óbito fetal e sobre o nascimento da criança. As entrevistas foram realizadas entre seis e 
dezoito meses após o parto e analisadas através de metodologia fenomenológica. As mulheres mencionaram que, durante a indução do parto, emergiram dificuldades em expressar suas preocupações, desejo de dar à luz imediatamente e certa sensação de estar "fora" da realidade. Referem também que não se sentiram respeitadas como seres humanos durante o processo de diagnóstico de morte intrauterina. Destacam ainda que os profissionais de saúde não devem apressar o processo de indução do parto e realizá-lo imediatamente após o diagnóstico de óbito fetal. Os autores do estudo reforçam a necessidade de os profissionais de saúde aproveitarem melhor o período que vai do diagnóstico de morte uterina até a indução do parto, para melhorar a capacidade das mulheres de elaborar o luto pela perda do bebê e prepará-las adequadamente para conhecê-lo e despedir-se dele. Os autores sugerem mais pesquisas para identificar os mecanismos de suporte aos pais e também os efeitos iatrogênicos do trauma psicológico que possam ser eliminados.

Uma pesquisa qualitativa realizada no Brasil por Santos, Rosenburg e Buralli (2004) relata a experiência de sete mulheres que vivenciaram a perda fetal. As mulheres foram selecionadas a partir de atestados de óbito de nascidos mortos, obtidos em cartório no período de um ano de estudo. Utilizaramse as técnicas de história oral para coleta de dados e de análise de conteúdo para compreensão dos resultados. O material analisado foi dividido em dois momentos posteriores à análise. $\mathrm{O}$ primeiro momento compreendeu a gestação e o impacto da perda, incluindo-se as seguintes categorias de analise: percepção da gestação, a chegada do bebê, os problemas de saúde e o atendimento recebido; o segundo momento incluiu o significado, para as mulheres, da perda ocorrida, relacionando-se as seguintes categorias: perda de parte do corpo, fatalidade relacionada a questões religiosas e mudanças de atitudes. As participantes relatam o desejo de viver, trabalhar, estudar e ter uma nova gestação. A rede de apoio social constituiu-se de apoio vindo da família e da Igreja. O apoio vindo dos serviços de saúde foi praticamente relatado como inexistente. Os autores do artigo enfatizam a necessidade de humanização do atendimento existente nos serviços de saúde, com o oferecimento de apoio multiprofissional às mulheres que passaram pela experiência de perda fetal, bem como reforçam a importância de uma rede de apoio consistente e atuante.

Resta ainda a apresentação de um artigo de Capitulo (2004). Esse artigo descreve uma pesquisa etnográfica realizada pela internet. Foram pesquisados 447 e-mails de 80 a 87 participantes de uma lista de mulheres enlutadas foram analisados. As mulheres da lista experienciaram abortamento e perda fetal ou neonatal. Os resultados do estudo foram descritos em onze categorias. A autora do artigo conclui que essa lista on-line cria uma cultura e uma comunidade específicas. Assim sendo, virtualmente, as mulheres podem expressar seus sentimentos em relação à perda de suas crianças e perceber que não são as únicas a vivenciar esse momento, visto que a internet possibilita a troca de experiências entre mulheres de diferentes locais e de diferentes culturas. Entre outras contribuições relevantes, o artigo destaca dois momentos aos quais a equipe de saúde deve se ater: a relevância das datas comemorativas na exacerbação do luto e a importância de rituais no momento de enlutamento.

\section{CONCLUSÃo}

Ao relatarem as experiências de mulheres que sofreram uma perda fetal, renomados autores de referência e dados dos artigos levantados em periódicos nos mostram a existência de sentimentos com os quais é difícil lidar, pois trazem à tona conflitos relacionados à identidade feminina e ao papel da mulher na sociedade. Trazem também para o centro de discussão a questão morte e seus inerentes tabus. A importância de se conhecer a dinâmica emocional das mulheres que vivenciaram a experiência de perda também está evidenciada. A equipe de saúde que estiver atenta a essa questão torna-se capaz de atender adequadamente à demanda emocional de tais mulheres e assim possibilitar-lhes uma elaboração adequada do luto pela perda do bebê. Ademais, propiciam a abertura de espaços adequados para essas mulheres expressarem seus sentimentos dolorosos, seja mediante atendimento no próprio local onde haja profissionais de saúde capacitados, seja por meio de encaminhamento para serviços que tenham seguimentos psicológicos ou psicossociais.

\section{REFERÊNCIAS}

Andajani-Suthahjo, S. \& Manderson, L. (2004). Stillbirth, neonatal death and reproductive rights in Indonesia. Reproductive Health Matters: 12(24), 181-88.

Bartilotti M. R. M. B. (1998). Obstetrícia e Ginecologia: Urgências Psicológicas. Em: Angerami-Camon (org), V. A. Urgências psicológicas no Hospital. São Paulo: Pioneira. 
Capitulo, K. L. (2004). Perinatal Grief online. MCN AmJ Matern Child Nurs: 29(5), 305-11.

Defey, D., Diaz, J. R. L., Niñez, M. \& Terra, C. (1992). Duelo por un niño que muere antes de nacer: vivencias de los padres del equipo de salud. 2 ed. Montevideo: CLAP.

Fretts, R. C. (2005). Etiology and prevetions of stillbirth. American Journal of Obstetrics and Gynecology, 193, 1923-35.

Hsu M. T., Tseng, Y. F., Banks, J. M. \& Kuo, L. L. (2002). Transforming loss:Taiwanese women's adaptation to stillbirth. J Adv Nurs; 40(4),387-95.

Hsu, M. T., Tseng, Y. F., Banks, J. M., Kuo, L. L. (2004). Interpretations of stillbirth. J Adv Nurs; 47(4), 408-16.

Kennell, J. H. \& Klaus, M. H. T. (1992). Atendimento aos pais de um natimorto ou de um bebê que morre. In: Klaus, M.H.T., Kennell, J.H. Pais/bebês: a formação do apego. Porto Alegre:Artes Médicas.

Maldonado, M. T. (1982). Maternidade e paternidade: preparação com técnicas de grupo. São Paulo Rio de Janeiro: Atheneu.

Mariani Neto, C. \& Aquino, M. M. A. (2000). Óbito Fetal. Em: Neme, B. Obstetrícia Básica. 2 ed. São Paulo: Sarvier.
Quayle, J. (1997). Óbito Fetal e Anomalias Fetais: repercussões emocionais maternas. Em Zugaib, M., Tedesco, J. J. \& Quayle, J. Obstetrícia Psicossomática. São Paulo: Atheneu.

Santos, A. L. D., Rosenburg,C. P. \& Buralli, K. O. (2004). Histórias de perdas fetais contada por mulheres:estudo de análise qualitativa.Rev.Saúde Pública, 38(2), 268-76.

St John, A., Cooke, M. \& Goopy, S. (2006). Shrouds of silence: three women's stories of prenatal loss. Aust J Adv Nurs, 23(3), 8-12.

Soifer R. (1992). Psicologia da Gravidez, Parto e Puerpério. Porto Alegre:Artes Médicas.

Trulson, O. \& Radestad, I. (2004). The silent child-mothers before, during and after stillbirth. Birth, 31(3), 189-95.

Videla, M. \& Grieco, A. (1993). Parir y nacer en el hospital. 1 ed. Buenos Aires: Nueva Vison.
Endereço para correspondência :

Claudia Aparecida Marchetti Duarte. Rua : Ernani Pereira Lopes, 52, ap.52, CEP 13091-132,
Campinas SP, Brasil.E-mail: clauamduarte@ yahoo.com.br
Recebido em 18/03/2008 Aceito em 01/08/2008 